\title{
Clinical Characteristics and Outcomes of Hospitalized Patients With Heart Failure From the Large-Scale Japanese Registry Of Acute Decompensated Heart Failure (JROADHF)
}

\author{
Tomomi Ide, MD, PhD; Hidetaka Kaku, MD; Shouji Matsushima, MD, PhD; \\ Takeshi Tohyama, MD, PhD; Nobuyuki Enzan, MD; Kouta Funakoshi, MD; Yoko Sumita; \\ Michikazu Nakai, PhD; Kunihiro Nishimura, MD, PhD; Yoshihiro Miyamoto, MD, PhD; \\ Miyuki Tsuchihashi-Makaya, $\mathrm{PhD}$; Masaru Hatano, $\mathrm{MD}$, PhD; Issei Komuro, MD, PhD; \\ Hiroyuki Tsutsui, MD, PhD; the JROADHF Investigators
}

\begin{abstract}
Background: With aging population, the prevalence and incidence of heart failure (HF) have been increasing worldwide. However, the characteristics and outcomes of patients with HF in an era of aging are not well established in Japan.
\end{abstract}

\begin{abstract}
Methods and Results: The Japanese Registry Of Acute Decompensated Heart Failure (JROADHF), a retrospective, multicenter, nationwide registry, was designed to study the clinical characteristics and outcomes of patients hospitalized with HF throughout Japan in 2013. One hundred and twenty-eight hospitals were selected by cluster random sampling and 13,238 hospitalized patients with HF were identified by medical record review. Demographics, medical history, severity, treatment, and in-hospital and long-term outcome data were collected from the Diagnostic Procedure Combination and medical charts. Data were analyzed using univariate and multivariate logistic regression or Cox regression analysis. The mean age of registered patients was $78.0 \pm 12.5$ years and $52.8 \%$ were male. Elderly patients (age $>75$ years) accounted for $68.9 \%$, and HF with preserved ejection fraction (HFpEF) accounted for $45.1 \%$. Median length of hospital stay was 18 days and in-hospital mortality was $7.7 \%$. The median follow-up period was 4.3 years, and the incidence rates for cardiovascular death and rehospitalization for HF were 7.1 and 21.1 per 100 person-years, respectively.
\end{abstract}

Conclusions: A contemporary nationwide registry demonstrated that hospitalized HF patients were very elderly, HFpEF was common, and their prognosis was still poor in Japan.

Key Words: Aging population; Heart failure; Heart failure with preserved ejection fraction; Outcomes; Registry

$\mathbf{H}$ eart failure (HF) is increasing in prevalence and incidence in the aging population worldwide., ${ }^{1,2}$ Despite significant advances in pharmacological and non-pharmacological therapies over the past few decades, $\mathrm{HF}$ is still associated with high mortality and rehospitalization rates and contributes substantially to the health-care cost. ${ }^{3,4}$ Japan is entering the most advanced aging society in the world, which accelerates the HF pandemic. ${ }^{5-7}$ However, the characteristics and outcomes of patients with HF have not been well investigated based on nationwide sur- veillance in an era of aging populations in Japan.

The Japanese Registry Of All cardiac and vascular Diseases-the Japanese Diagnosis Procedure Combination system (JROAD-DPC) was developed as a nationwide claim database in 2014, using data from the Japanese Diagnosis Procedure Combination/Per Diem Payment System. JROAD-DPC uses codes based on diagnosis categories and diagnosis groups, and the following information is retrospectively collected for each patient: unique hospital identifier, age, sex, diagnoses, comorbidities, length

Received September 23, 2020; revised manuscript received January 30, 2021; accepted February 14, 2021; J-STAGE Advance Publication released online April 15, 2021 Time for primary review: 32 days

Department of Cardiovascular Medicine (T.I., H.K., S.M., T.T., N.E., K.F., H.T.), Division of Cardiovascular Medicine, Research Institute of Angiocardiology (T.I., H.K., H.T.), Faculty of Medical Sciences, Kyushu University, Fukuoka; Center for Clinical and Translational Research, Kyushu University Hospital, Fukuoka (T.T., K.F.); Center for Cerebral and Cardiovascular Disease Information, National Cerebral and Cardiovascular Center, Suita (Y.S., M.N., K.N., Y.M.); School of Nursing, Kitasato University, Sagamihara (M.T.-M); and Department of Cardiovascular Medicine, Graduate School of Medicine, The University of Tokyo, Tokyo (M.H., I.K.), Japan

Mailing address: Tomomi Ide, MD, PhD, Department of Cardiovascular Medicine, Faculty of Medical Sciences, Kyushu University, 3-1-1 Maidashi, Higashi-ku, Fukuoka 812-8582, Japan. E-mail: tomomi_i@cardiol.med.kyushu-u.ac.jp

All rights are reserved to the Japanese Circulation Society. For permissions, please e-mail: cj@j-circ.or.jp

ISSN-1346-9843 


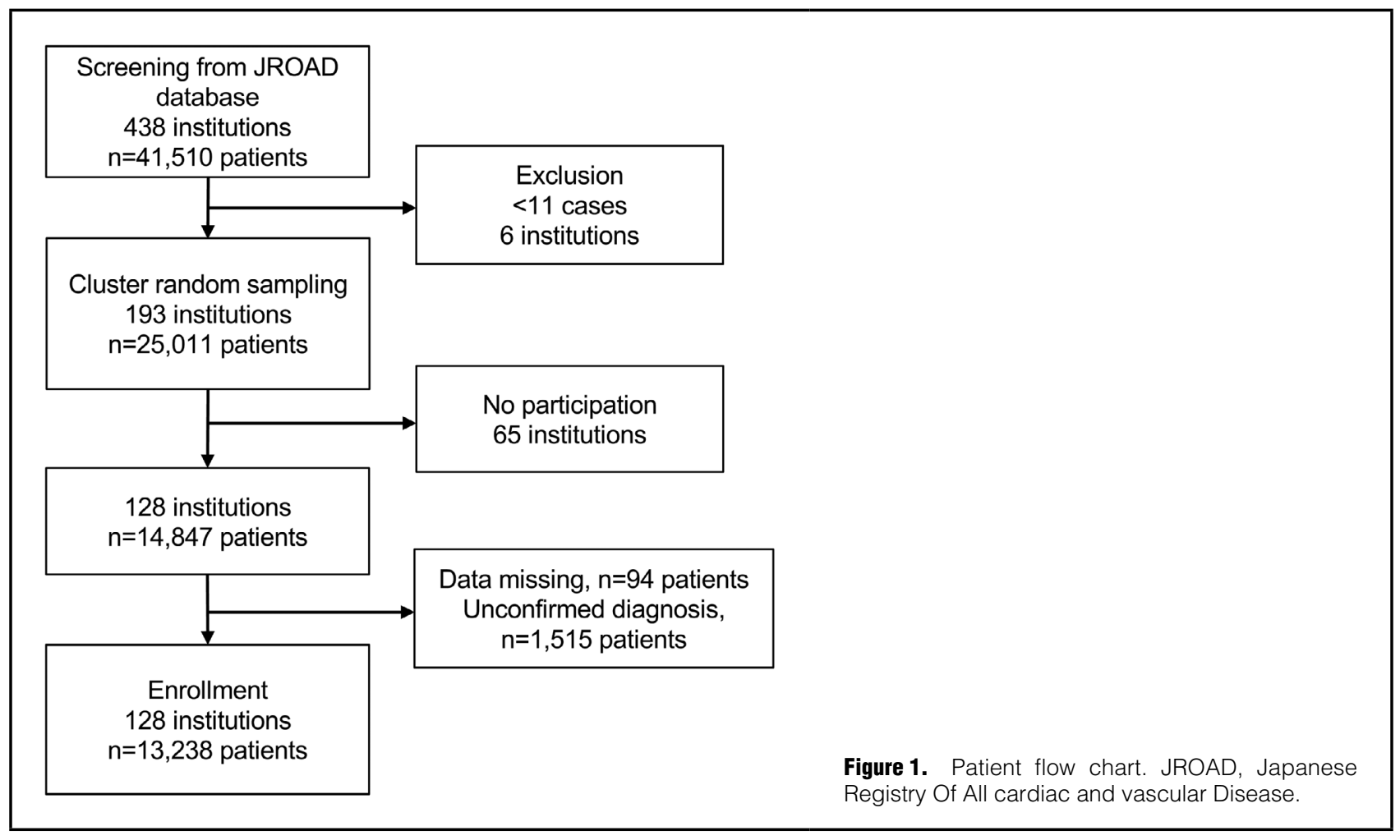

of stay, discharge status, drugs, devices, and therapeutic procedures. ${ }^{\mathbf{8}}$ The JROAD-DPC database provides various types of in-hospital data, although it lacks vital signs, laboratory data, echocardiographic findings, and long-term outcomes for each patient.

The Japanese Registry Of Acute Decompensated Heart Failure (JROADHF) is a retrospective, multicenter, nationwide registry of hospitalized patients with $\mathrm{HF}$. The aim of this registry is to provide a summary of contemporary features, management, and in-hospital as well as longterm outcome of hospitalized patients with HF across a large range of centers in Japan. The current study aimed to investigate the clinical characteristics and outcomes of hospitalized patients with HF from JROADHF.

\section{Methods}

\section{Study Setting}

The JROADHF database was developed in collaboration with the Japanese Circulation Society (JCS) and the Japanese Heart Failure Society. This registry was developed by linking the JROAD-DPC database with medical records, including long-term follow up after discharge, which was retrospectively obtained by board-certified cardiologists.

We first selected 193 institutions from eligible institutions having more than 10 acute $\mathrm{HF}$ admissions during 2013 by cluster random sampling according to a previous method. ${ }^{9}$ We collected DPC data directly from each hospital and created a list of patients with HF admission by combining International Classification of Diseases-10 diagnosis codes related to HF (I50.0, I50.1 and I50.9) and an additional diagnostic code (30101 or 30102 representing acute HF or exacerbation of chronic HF). The identification of the listed HF patients was conducted by the investigators in each hospital based on DPC number, which was unique for each patient and converted to the patients' identification number. Institutions were excluded if they were not willing to participate in this registry.

\section{Study Patient Database}

The following data were extracted from the JROAD-DPC database: unique hospital identifier, patient age, sex, diagnoses, medical procedures, in-hospital management, prescribed medications, discharge status, and medical costs. Causes of HF, comorbidities at admission, medical history, New York Heart Association (NYHA) functional class at admission and discharge, vital signs, echocardiographic parameters, laboratory data at admission and discharge, and long-term prognosis including any cause of death and cardiovascular hospitalization were adjudicated by the investigator of each institution based on patients' medical records. A follow-up survey was conducted until December 2017. The 1,515 patients who were not diagnosed with HF by physicians were excluded.

\section{Outcomes}

The primary outcome of this study was all-cause death during hospitalization and all-cause death during the follow-up period. Secondary outcomes were in-hospital death due to myocardial infarction, HF, arrhythmia, stroke, pulmonary embolism, pneumonia, and malignancy. Cardiovascular death, HF hospitalization, and composite of cardiovascular death or HF hospitalization during the follow-up period were also assessed.

\section{Comparison With Other Registries}

Baseline characteristics, in-hospital interventions, medication at discharge, length of hospital stay, and in-hospital death were compared with previous registries including JCARE-CARD, ${ }^{10}$ acute decompensated heart failure 


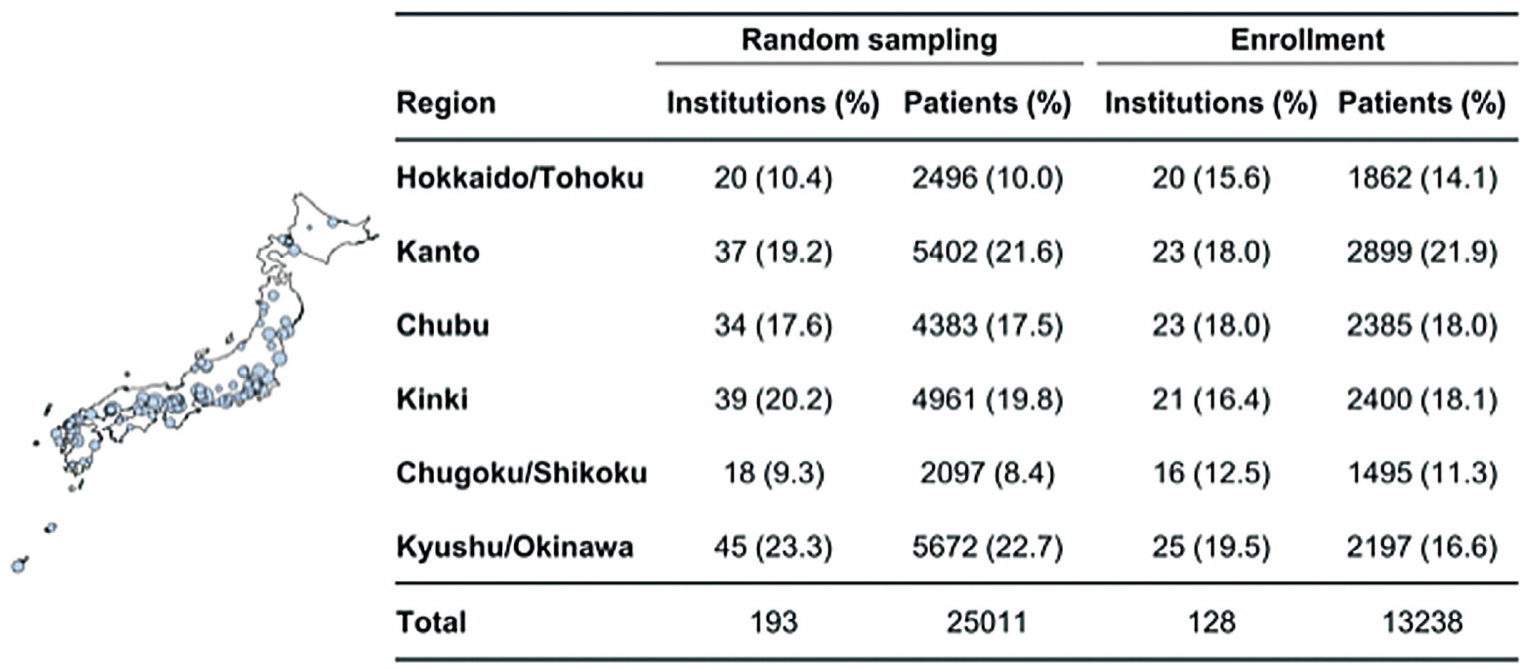

Figure 2. Nationwide distribution of participating institutions in the Japanese Registry Of Acute Decompensated Heart Failure (JROADHF).

syndrome (ATTEND), ${ }^{11}$ West Tokyo-Heart Failure (WET$\mathrm{HF}),{ }^{12}$ and the European Society of Cardiology heart failure long-term registry (ESC-HF-LT). ${ }^{3,13}$

\section{Statistical Analysis}

Continuous variables are presented as mean \pm standard deviation when normally distributed or otherwise as median and interquartile range (IQR). There are some missing data and we conducted complete case analysis. Odds ratio (OR) were estimated using a logistic regression model and presented with $95 \%$ confidence intervals (CIs) and $\mathrm{P}$ values. We included covariates that were statistically significant in the univariate logistic regression analysis of factors associated with in-hospital mortality in a multivariate logistic regression model. The same variable selection method was used in the Cox regression model for cardiovascular death during the follow-up period. All tests were 2-tailed, and a $\mathrm{P}$ value $<0.05$ was considered statistically significant. The analyses were performed using $\mathrm{SAS}^{\circledR 9} 9.4$ (SAS Institute Inc., Cary, NC, USA).

\section{Ethics Statement}

This study was conducted in accordance with the principles of the Declaration of Helsinki. The use of the JROADDPC database for the initial calculation was approved by the ethical committee of the JCS (2017. No3). The study protocol using the JROADHF database was approved by Kyushu University (2019-569) and all 128 participating hospitals. The requirement for individual informed consent based on the "opt-out" principle was applied because of the retrospective observational design of the present study. Each hospital anonymized patients' ID using codechange equations, which were applied to the original DPC data, and patients were notified that their information was collected by this study through homepages or posters in each hospital and on the study website (https://www. jroadhf.jp). Patients could choose to have their information excluded. The authors had full access to and take full responsibility for the integrity of the data.

\section{Results}

\section{Eligible Institutions and Patients}

Out of 438 institutions participating in the JROAD-DPC registry, 6 institutions were excluded because they had $\leq 10$ hospitalized patients with HF (Figure 1). We selected 193 institutions by cluster random sampling. Of these, 128 institutions agreed to participate in the JROADHF registry. JROADHF investigators confirmed the diagnosis of hospitalization due to $\mathrm{HF}$ exacerbation among 13,238 out of 14,847 patients based on medical record review. The participating institutions and patients were distributed nationwide similarly before and after screening (Figure 2).

\section{Baseline Characteristics of Studied Patients}

The mean age of patients was $78.0 \pm 12.5$ years and $52.8 \%$ were male (Table 1). Female patients were older than male patients $(81.6 \pm 11.1$ vs. $74.8 \pm 12.8$ years, $\mathrm{P}<0.001)$ (Supplementary Figure). The major etiologies of hospitalized patients with $\mathrm{HF}$ were ischemic in $26.6 \%$, valvular in $18.5 \%$, arrhythmic in $17.3 \%$, hypertensive in $16.5 \%$, and cardiomyopathic in $12.4 \%$. The comorbidities included hypertension in $71.2 \%$, valvular heart disease in $35.3 \%$ (mitral valve regurgitation $46.4 \%$, aortic valve stenosis $25.3 \%$, and others $28.3 \%$ ), diabetes mellitus in $34.2 \%$, dyslipidemia in $30.1 \%$, chronic kidney disease in $38.9 \%$, anemia in $21.2 \%$, stroke in $13.7 \%$, and chronic obstructive pulmonary disease in $6.4 \%$ of patients. Patients with a prior history of $\mathrm{HF}$ admission accounted for $34.7 \%$ of the cohort. Those with HF with reduced ejection fraction (HFrEF: LVEF $<40 \%$ ), HF with mid-range ejection fraction (HFmrEF: LVEF 40-49\%), and HF with preserved ejection fraction (HFpEF: LVEF $\geq 50 \%$ ) accounted for $37.4 \%, 17.5 \%$, and $45.1 \%$ of the cohort, respectively. HFpEF was more prevalent in female patients $(58.3 \%)$ than in male patients. During hospitalization, $79.5 \%$ of patients were treated with oxygen, and a cardiac rehabilitation program was undertaken by $29.1 \%$ of patients. Diuretics were prescribed for $90.1 \%$ of patients at discharge. Inhibi- 
Table 1. Baseline Characteristics of Study Patients

\section{Characteristics}

$\mathrm{n}=\mathbf{1 3 , 2 3 8}$

Age, years

$78.0 \pm 12.5$

Male, \%

$6,991(52.8)$

BMI, $k g / m^{2}$ ( $n=11,945$, missing $\left.n=1,293\right)$

$22.9+4.6$

Prior hospitalization due to heart failure, \% $(n=13,238)$

$4,599(34.7)$

Etiology of heart failure, $\%(n=12,763$, missing $n=475)$

Ischemic

3,401 (26.6)

Valvular

$2,365(18.5)$

Arrhythmia

$2,202(17.3)$

Hypertensive

$2,102(16.5)$

Cardiomyopathic

$1,587(12.4)$

Others

436 (3.4)

Unknown

534 (4.2)

Comorbidities, \% $(n=13,238)$

Hypertension

$9,420(71.2)$

Ischemic heart disease

4,701 (35.5)

Valvular heart disease

4,671 (35.3)

Diabetes mellitus

$4,534(34.2)$

Dyslipidemia

$3,990(30.1)$

CKD

$5,152(38.9)$

Hyperuricemia

$2,848(21.5)$

Anemia

$2,804(21.2)$

Stroke

$1,816(13.7)$

PAD

$788(6.0)$

COPD

846 (6.4)

Sleep-disordered breathing

$508(3.8)$

Medical history, \%

$\mathrm{PCl}$

CABG

Supraventricular arrhythmia

Atrial fibrillation

Others

Ablation

Pacemaker

VT/VF

ICD

CRT-P

CRT-D

Valve surgery

Hemodialysis

Cardiac rehabilitation

Vital signs at admission

SBP, $\mathrm{mmHg}$

DBP, $\mathbf{m m H g}$

Pulse rate, bpm

NYHA functional class, $\%(n=12,999$, missing $n=239)$

I-II

III-IV

Echocardiographic parameters at admission

( $n=11,573$, missing $n=1,665)$

LVEF, \%

HFrEF, \%

HFmrEF, \%

HFpEF, \% $2,250(17.5)(n=12,874$, missing $n=364)$

$870(6.8)(n=12,874$, missing $n=364)$

$5,677(43.1)(n=13,175$, missing $n=63)$

505 (3.9) $(n=12,867$, missing $n=371)$

$204(1.6)(n=12,874$, missing $n=364)$

$944(7.7)(n=12,236$, missing $n=1,002)$

$561(4.4)(n=12,860$, missing $n=378)$

$163(1.3)(n=12,188$, missing $n=1,050)$

$53(0.4)(n=12,190$, missing $n=1,048)$

$246(2.0)(n=12,178$, missing $n=1,060)$

$725(6.0)(n=12,184$, missing $n=1,054)$

$298(2.4)(n=12,175$, missing $n=1,063)$

469 (3.9) $(n=12,174$, missing $n=1,064)$

$139.9 \pm 33.0(n=13,102$, missing $n=136)$

$79.7 \pm 21.6(n=13,029$, missing $n=209)$

$91.9 \pm 25.9(n=13,045$, missing $n=193)$

$1,929(14.8)$

$11,070(85.2)$

$46.7 \pm 17.3$

$4,329(37.4)$

$2,024(17.5)$

$5,220(45.1)$

(Table 1 continued the next page.) 


\begin{tabular}{|c|c|}
\hline Characteristics & $n=13,238$ \\
\hline \multicolumn{2}{|l|}{ Laboratory data at admission } \\
\hline Hemoglobin, g/dL & $11.6 \pm 2.4(n=13,115$, missing $n=123)$ \\
\hline Albumin, g/dL & $3.5 \pm 0.6(n=11,708$, missing $n=1,530)$ \\
\hline BUN, mg/dL & $23.9(17.4-34.7)(n=13,150$, missing $n=88)$ \\
\hline Creatinine, mg/dL & $1.1(0.8-1.6)(n=13,190$, missing $n=48)$ \\
\hline eGFR, $\mathrm{mL} / \mathrm{min} / 1.73 \mathrm{~m}^{2}$ & $46.7 \pm 24.7(n=13,190$, missing $n=48)$ \\
\hline Sodium, $\mathrm{mEq} / \mathrm{L}$ & $139.2 \pm 4.8(n=13,183$, missing $n=55)$ \\
\hline Potassium, mEq/L & $4.3 \pm 0.7(n=13,175$, missing $n=63)$ \\
\hline $\mathrm{BNP}, \mathrm{pg} / \mathrm{mL}$ & $692.6(375.7-1,309.0)(n=9,688$, missing $n=3,550)$ \\
\hline \multicolumn{2}{|c|}{ Medication at admission $(n=13,232-13,233$, missing $n=5-6)$} \\
\hline Diuretics & $7,258(54.9)$ \\
\hline ACEI & $1,868(14.1)$ \\
\hline ARB & $4,439(33.5)$ \\
\hline ACEI or ARB & $6,119(46.2)$ \\
\hline$\beta$-blockers & $4,805(36.3)$ \\
\hline MRA & $2,859(21.6)$ \\
\hline \multicolumn{2}{|l|}{ In-hospital management, $\%(n=12,446$, missing $n=792)$} \\
\hline Oxygen inhalation & $9,898(79.5)$ \\
\hline Mechanical ventilation with intubation & $377(3.0)$ \\
\hline Mechanical ventilation without intubation & $1,978(15.9)$ \\
\hline Vasodilator & $8,032(64.5)$ \\
\hline Inotrope & $2,634(21.2)$ \\
\hline IABP & $96(0.8)$ \\
\hline PCPS & $13(0.1)$ \\
\hline Cardiac rehabilitation & $3,618(29.1)$ \\
\hline \multicolumn{2}{|l|}{ Medication at discharge, $\% *(n=10,753$, missing $n=367)$} \\
\hline Diuretics & $9,691(90.1)$ \\
\hline ACEI & $3,238(30.1)$ \\
\hline ARB & $4,194(39.0)$ \\
\hline ACEI or ARB & $7,087(65.9)$ \\
\hline$\beta$-blockers & $6,855(63.7)$ \\
\hline MRA & $5,476(50.9)$ \\
\hline Transfer to hospital or nursing facility, $\%(n=11,120)$ & $1,231(11.1)$ \\
\hline
\end{tabular}

Data are shown as $n(\%)$, means $\pm S D$, or median (IQR), unless specified otherwise. ACEI, angiotensin-converting enzyme inhibitor; ARB, angiotensin II receptor blocker; BMI, body mass index; BNP, brain-type natriuretic peptide; BUN, blood urea nitrogen; CABG, coronary artery bypass grafting; CKD, chronic kidney disease; COPD, chronic obstructive pulmonary disease; CRT-D, cardiac resynchronization therapy defibrillator; CRT-P, cardiac resynchronization therapy pacemaker; DBP, diastolic blood pressure; eGFR, estimated glomerular filtration rate; HFmrEF, heart failure with mid-range ejection fraction; HFpEF, heart failure with preserved ejection fraction; HFrEF, heart failure with reduced ejection fraction; IABP, intra-aortic balloon pumping; ICD, implantable cardioverter defibrillator; LVEF, left ventricular ejection fraction; MRA, mineralocorticoid receptor antagonist; NYHA, New York Heart Association functional classification; $P A D$, peripheral artery disease; $P C I$, percutaneous coronary intervention; PCPS, percutaneous cardio-pulmonary support; SBP, systolic blood pressure; VF, ventricular fibrillation; VT, ventricular tachycardia.

tors of the renin-angiotensin system (RAS), particularly angiotensin-converting enzyme inhibitors (ACEIs) and angiotensin II receptor blockers (ARBs), and $\beta$-blockers were prescribed at discharge for $65.9 \%$ and $63.7 \%$ of patients, respectively. The patients who were prescribed both RAS inhibitors and $\beta$-blockers were $46.2 \%$.

\section{In-Hospital Death}

The median duration of hospital stay was 18 (IQR 12-28) days. The in-hospital mortality rate was $7.7 \%$, with cardiovascular death and non-cardiovascular death accounting for $77.1 \%$ and $20.9 \%$ of those patients, respectively (Table 2). In particular, $66.2 \%$ of patients died of HF. Among causes of non-cardiovascular deaths, pneumonia was the most common cause of death, accounting for $9.1 \%$. The multivariable logistic analysis showed that age
(OR 1.56; 95\% CI 1.38-1.75; P<0.001), NYHA functional class (OR 3.85; 95\% CI 2.33-6.36; $\mathrm{P}<0.001$ ), aortic valve stenosis (OR 1.67; 95\% CI 1.25-2.22; $\mathrm{P}<0.001$ ), chronic kidney disease stage IV-V (OR 2.02; 95\% CI 1.60-2.55; $\mathrm{P}<0.001$ ), stroke (OR 1.45; 95\% CI 1.12-1.88; $\mathrm{P}=0.005$ ), and log-transformed brain natriuretic peptide (BNP) level (OR 1.57; 95\% CI $1.39-1.79 ; \mathrm{P}<0.001$ ) were positively associated with in-hospital death, and systolic blood pressure (OR 0.89; 95\% CI 0.85-0.92; $\mathrm{P}<0.001)$, hypertension (OR 0.67; 95\% CI 0.53-0.84; P<0.001), dyslipidemia (OR $0.62 ; 95 \%$ CI $0.48-0.82 ; \mathrm{P}<0.001)$, serum albumin at admission (OR 0.45; 95\% CI 0.37-0.55; $\mathrm{P}<0.001$ ), serum sodium at admission (OR 0.97 ; 95\% CI 0.95-0.98; $\mathrm{P}<0.001$ ), and $\beta$-blocker use at admission (OR 0.71; 95\% CI $0.56-0.90 ; \mathrm{P}=0.005)$ were negatively associated with in-hospital death (Table 3). 


\section{Long-Term Outcomes}

We investigated long-term outcomes in 11,120 patients after discharge. Among those patients discharged, 1,231 patients $(11.1 \%$, missing $n=368)$ were transferred to hospitals or a nursing facility. The median follow-up period was 4.3 (IQR 3.8-4.7) years. The follow-up rates were $91 \%$ at 1 year and $80 \%$ at 3 years after discharge. The incidence rates (100 person-years) were 24.1 for composite of cardiovascular death or HF hospitalization, 7.1 for cardiovascular death, 21.1 for HF hospitalization, and 14.9 for all-cause death (Supplementary Table). Event-free survival rates at 4 years after discharge was $46.2 \%$ (95\% CI $45.1-$ $47.2 \%$ ) for the composite of cardiovascular death or HF hospitalization, $75.9 \%$ (95\% CI 75.0-76.8\%) for cardiovascular death, $51.6 \%(95 \%$ CI $50.5-52.7 \%)$ for HF hospitalization, and $55.7 \%(95 \%$ CI $54.7-56.7 \%)$ for all-cause death (Figure 3A-D). The same rates at 4 years after admission were $43.6 \%(95 \%$ CI $42.6-44.7 \%)$ for the composite of cardiovascular death or HF hospitalization, 71.4\% (95\% CI $70.5-72.3 \%)$ for cardiovascular death, $52.2 \%(95 \%$ CI $51.0-53.3 \%)$ for HF hospitalization, and $51.6 \%(95 \%$ CI $50.6-52.5 \%$ ) for all-cause death. The multivariable Cox regression analysis showed that age (hazard ratio [HR]
$1.43 ; 95 \%$ CI 1.35-1.50; $\mathrm{P}<0.001)$, NYHA functional class (HR 1.36; 95\% CI 1.18-1.57; P<0.001), prior HF hospitalization (HR 1.63; 95\% CI 1.48-1.80; $\mathrm{P}<0.001$ ), ischemic heart disease (HR 1.16; 95\% CI 1.01-1.32; $\mathrm{P}=0.03$ ), aortic valve stenosis (HR 1.66; 95\% CI 1.45-1.91; $\mathrm{P}<0.001$ ),

\begin{tabular}{|lr|}
\hline \multicolumn{1}{|c|}{ Table 2. Causes } & \\
In-hospital death & $\mathbf{n}=\mathbf{1 3 , 2 3 8}$ \\
Cardiovascular death & $1,023(7.7)$ \\
Myocardial infarction & $789(6.0)$ \\
Heart failure & $33(0.3)$ \\
Arrhythmia & $677(5.1)$ \\
Stroke & $48(0.4)$ \\
Others & $9(0.1)$ \\
Non-cardiovascular death & $22(0.2)$ \\
Pneumonia & $214(1.6)$ \\
Malignancy & $93(0.7)$ \\
Others & $25(0.2)$ \\
\hline
\end{tabular}

Data are presented as $\mathrm{n}(\%)$.

\begin{tabular}{|c|c|c|c|c|}
\hline \multirow{2}{*}{ Variables } & \multicolumn{2}{|c|}{ Univariate analysis } & \multicolumn{2}{|c|}{ Multivariate analysis } \\
\hline & OR (95\% Cl) & $P$ value & OR (95\% Cl) & $P$ value \\
\hline Age (decade) & $1.65(1.54-1.77)$ & $<0.001$ & $1.56(1.38-1.75)$ & $<0.001$ \\
\hline Female & $1.31(1.15-1.49)$ & $<0.001$ & $1.04(0.83-1.30)$ & 0.73 \\
\hline $\mathrm{SBP}, 10 \mathrm{mmHg}$ & $0.83(0.81-0.85)$ & $<0.001$ & $0.89(0.85-0.92)$ & $<0.001$ \\
\hline Pulse rate, $10 \mathrm{mmHg}$ & $0.98(0.95-1.00)$ & 0.059 & & \\
\hline NYHA class (III/IV) & $3.60(2.68-4.83)$ & $<0.001$ & $3.85(2.33-6.36)$ & $<0.001$ \\
\hline Prior HF hospitalization & $1.38(1.21-1.57)$ & $<0.001$ & $1.20(0.95-1.51)$ & 0.12 \\
\hline Ischemic heart disease & $1.08(0.95-1.23)$ & 0.26 & & \\
\hline Aortic valve stenosis & $1.93(1.61-2.32)$ & $<0.001$ & $1.67(1.25-2.22)$ & $<0.001$ \\
\hline Hypertension & $0.51(0.44-0.58)$ & $<0.001$ & $0.67(0.53-0.84)$ & $<0.001$ \\
\hline Diabetes mellitus & $0.79(0.69-0.91)$ & $<0.001$ & $0.99(0.78-1.26)$ & 0.96 \\
\hline Dyslipidemia & $0.50(0.43-0.59)$ & $<0.001$ & $0.62(0.48-0.82)$ & $<0.001$ \\
\hline CKD stage IV-V & $2.58(2.27-2.94)$ & $<0.001$ & $2.02(1.60-2.55)$ & $<0.001$ \\
\hline Stroke & $1.45(1.23-1.72)$ & $<0.001$ & $1.45(1.12-1.88)$ & 0.005 \\
\hline LVEF $<40 \%$ & $1.28(1.10-1.49)$ & 0.001 & $1.15(0.90-1.46)$ & 0.26 \\
\hline Atrial fibrillation & $0.83(0.72-0.94)$ & 0.004 & $0.95(0.77-1.19)$ & 0.67 \\
\hline VT/VF & $0.89(0.64-1.24)$ & 0.49 & & \\
\hline Coronary revascularization & $0.76(0.64-0.90)$ & 0.002 & $0.80(0.60-1.06)$ & 0.12 \\
\hline Pacemaker & $1.11(0.87-1.41)$ & 0.41 & & \\
\hline ICD & $0.88(0.48-1.63)$ & 0.69 & & \\
\hline CRT-P & $0.73(0.23-2.34)$ & 0.60 & & \\
\hline CRT-D & $0.84(0.51-1.41)$ & 0.51 & & \\
\hline Hemodialysis & $1.07(0.70-1.63)$ & 0.77 & & \\
\hline Hemoglobin, g/dL & $0.85(0.83-0.88)$ & $<0.001$ & $1.01(0.96-1.07)$ & 0.63 \\
\hline Albumin, $\mathrm{g} / \mathrm{dL}$ & $0.33(0.29-0.37)$ & $<0.001$ & $0.45(0.37-0.55)$ & $<0.001$ \\
\hline Sodium, mEq/L & $0.95(0.93-0.96)$ & $<0.001$ & $0.97(0.95-0.98)$ & $<0.001$ \\
\hline Potassium, mEq/L & $1.65(1.52-1.79)$ & $<0.001$ & $1.20(1.04-1.39)$ & 0.01 \\
\hline $\ln (B N P)$ & $1.75(1.60-1.92)$ & $<0.001$ & $1.57(1.39-1.79)$ & $<0.001$ \\
\hline Diuretics use at admission & $1.29(1.13-1.47)$ & $<0.001$ & $1.15(0.90-1.47)$ & 0.28 \\
\hline ACEI or ARB use at admission & $0.68(0.60-0.78)$ & $<0.001$ & $0.91(0.73-1.13)$ & 0.40 \\
\hline$\beta$-blocker use at admission & $0.68(0.59-0.78)$ & $<0.001$ & $0.71(0.56-0.90)$ & 0.005 \\
\hline MRA use at admission & $1.26(1.08-1.46)$ & 0.002 & $1.27(0.99-1.64)$ & 0.06 \\
\hline
\end{tabular}

OR, odds ratio; $\mathrm{Cl}$, confidence interval. Other abbreviations as in Table 1. 


\section{A. All cause death}

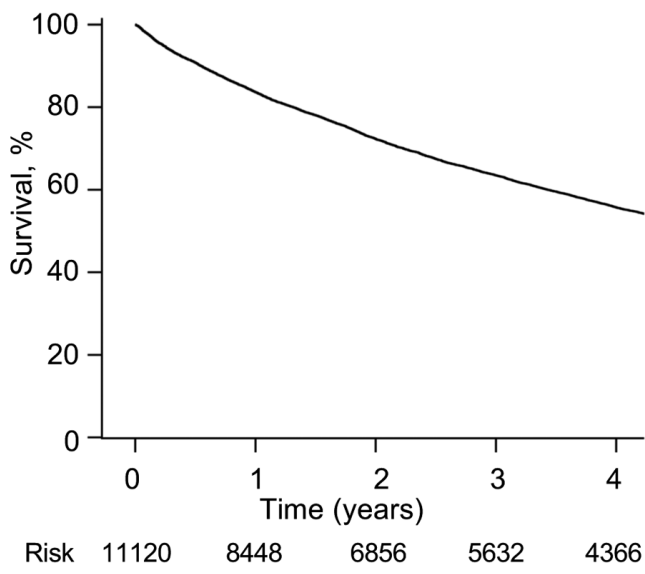

C. HF hospitalization

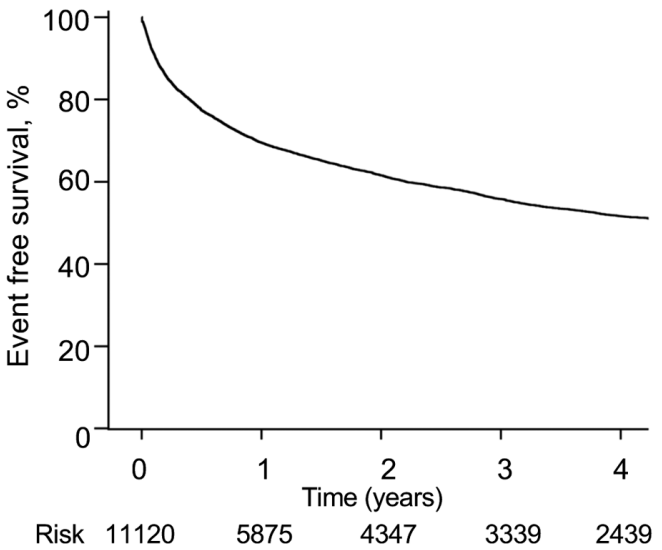

B. Cardiovascular death

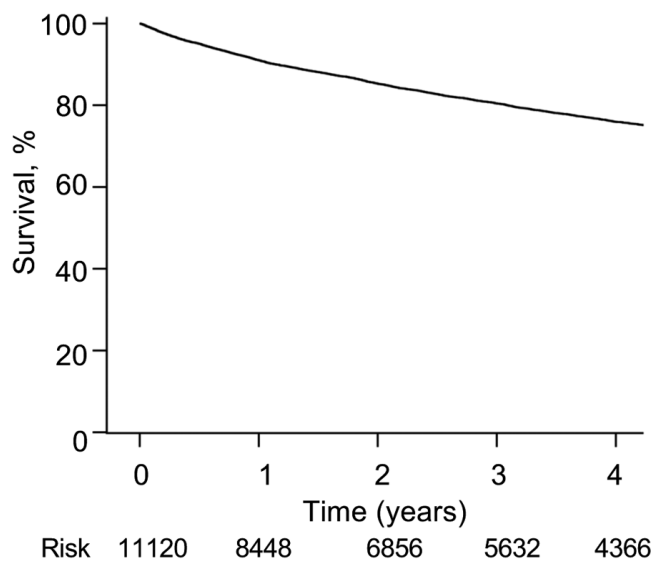

D. Cardiovascular death and HF hospitalization

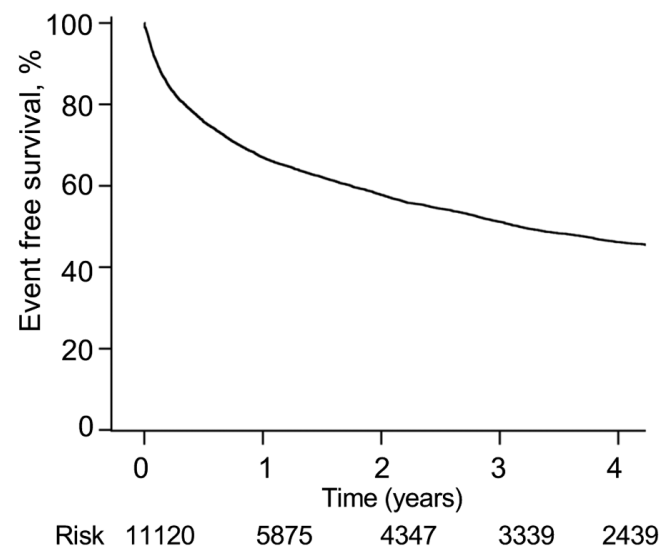

Figure 3. Kaplan-Meier curves for each outcomes after discharge. (A) All cause death, (B) Cardiovascular death, (C) HF hospitalization, (D) Cardiovascular death and HF hospitalization. HF, heart failure.

chronic kidney disease stage $4-5$ (HR $1.57 ; 95 \%$ CI $1.41-$ $1.75 ; \mathrm{P}<0.001)$, ventricular tachycardia/ventricular fibrillation (HR 1.32; 95\% CI 1.08-1.63; P=0.007), cardiac resynchronization therapy-defibrillator (CRT-D) (HR 1.61; 95\% CI 1.27-2.05; $\mathrm{P}<0.001)$, diuretic use at discharge (HR $1.28 ; 95 \%$ CI $1.06-1.55 ; \mathrm{P}=0.009)$, and transfer to hospital or a nursing facility at discharge (HR $1.31 ; 95 \%$ CI 1.14 $1.51 ; \mathrm{P}<0.001)$ were positively associated with cardiovascular death during follow up, and female sex (HR 0.82; 95\% CI 0.74-0.90; $\mathrm{P}<0.001$ ), systolic blood pressure (HR 0.92; 95\% CI 0.91-0.94; $\mathrm{P}<0.001$ ), hypertension (HR 0.87; 95\% CI 0.78-0.97; $\mathrm{P}=0.01$ ), hemoglobin (HR 0.91; 95\% CI 0.89-0.93; $\mathrm{P}<0.001$ ), sodium (HR 0.95; 95\% CI 0.94-0.96; $\mathrm{P}<0.001$ ), potassium (HR 0.82; 95\% CI 0.75-0.89; $\mathrm{P}<0.001$ ), ACEI/ARB use at discharge (HR 0.82; 95\% CI 0.74-0.91; $\mathrm{P}<0.001)$ were negatively associated with cardiovascular death during the follow-up period (Table 4).

\section{Comparison With Previous Heart Failure Registries}

Patients registered in JROADHF in the present study were older $(78.0 \pm 12.5$ years) than those in JCARE-CARD
(70.7 \pm 13.5 years), ATTEND (73.0 \pm 13.8 years), WET-HF (75.0 \pm 13.0 years), and the ESC-HF long-term registry (ESC-HF-LT) $(69.4 \pm 13.0$ years) (Table 5). Female patients with HF were more prevalent in JROADHF (47.2\%) than in JCARE-CARD $(40.6 \%)$, ATTEND $(42.0 \%)$, and WETHF $(41.0 \%)$. Mean left ventricular ejection fraction (LVEF) was higher in JROADHF $(46.7 \pm 17.3 \%)$ than in JCARE-CARD (42.5 $\pm 17.9 \%)$, WET-HF $(45.0 \pm 15.0 \%)$, and ESC-HF-LT $(39.2 \pm 14.5 \%)$. ACEI or ARBs were prescribed to a lesser extent at discharge for patients in JROADHF (65.6\%) than in JCARE-CARD (79.0\%) and ESC-HF-LT (77.0\%). $\beta$-blockers use was also lower for patients in JROADHF $(63.0 \%)$ than in ESC-HF-LT $(72.6 \%)$ and ATTEND (67.4\%), but it was higher than JCARE-CARD (49.8\%). The in-hospital mortality rate was higher for patients in JROADHF $(7.7 \%)$ than in ATTEND (6.4\%), WET-HF (4.7\%), and ESC-HF-LT $(5.5 \%)$. In-hospital all-cause and cardiac deaths were higher for patients in JROADHF than in other registries, ATTEND, WET-HF, and ESC-HF-LT. The 1-year allcause mortality rate was also higher for patients in 


\begin{tabular}{|c|c|c|c|c|}
\hline \multirow{2}{*}{ Variables } & \multicolumn{2}{|c|}{ Univariate analysis } & \multicolumn{2}{|c|}{ Multivariate analysis } \\
\hline & HR $(95 \% \mathrm{Cl})$ & $P$ value & HR (95\% Cl) & $P$ value \\
\hline Age (decade) & $1.53(1.47-1.59)$ & $<0.001$ & $1.43(1.35-1.50)$ & $<0.001$ \\
\hline Female & $1.15(1.05-1.25)$ & 0.001 & $0.82(0.74-0.90)$ & $<0.001$ \\
\hline $\mathrm{SBP}, 10 \mathrm{mmHg}$ & $0.91(0.89-0.92)$ & $<0.001$ & $0.92(0.91-0.94)$ & $<0.001$ \\
\hline Pulse rate, $10 \mathrm{mmHg}$ & $0.93(0.91-0.94)$ & $<0.001$ & $1.01(0.99-1.03)$ & 0.48 \\
\hline NYHA class (III/IV) & $1.88(1.66-2.14)$ & $<0.001$ & $1.36(1.18-1.57)$ & $<0.001$ \\
\hline Prior HF hospitalization & $2.10(1.93-2.28)$ & $<0.001$ & $1.63(1.48-1.80)$ & $<0.001$ \\
\hline Ischemic heart disease & $1.20(1.11-1.31)$ & $<0.001$ & $1.16(1.01-1.32)$ & 0.03 \\
\hline Aortic valve stenosis & $2.01(1.77-2.27)$ & $<0.001$ & $1.66(1.45-1.91)$ & $<0.001$ \\
\hline Hypertension & $0.83(0.76-0.91)$ & $<0.001$ & $0.87(0.78-0.97)$ & 0.01 \\
\hline Diabetes mellitus & $0.87(0.80-0.95)$ & 0.002 & $0.91(0.82-1.01)$ & 0.06 \\
\hline Dyslipidemia & $0.86(0.78-0.94)$ & $<0.001$ & $0.92(0.82-1.02)$ & 0.11 \\
\hline CKD stage IV-V & $2.01(1.84-2.19)$ & $<0.001$ & $1.57(1.41-1.75)$ & $<0.001$ \\
\hline Stroke & $1.24(1.10-1.39)$ & $<0.001$ & $1.04(0.91-1.19)$ & 0.56 \\
\hline Atrial fibrillation & $1.02(0.94-1.12)$ & 0.57 & & \\
\hline VT/VF & $1.64(1.40-1.93)$ & $<0.001$ & $1.32(1.08-1.63)$ & 0.007 \\
\hline Coronary revascularization & $1.26(1.14-1.38)$ & $<0.001$ & $1.04(0.89-1.21)$ & 0.63 \\
\hline Pacemaker & $1.34(1.17-1.54)$ & $<0.001$ & $1.00(0.85-1.17)$ & 0.99 \\
\hline ICD & $1.57(1.17-2.09)$ & 0.002 & $1.16(0.83-1.63)$ & 0.38 \\
\hline CRT-P & $1.98(1.36-2.90)$ & $<0.001$ & $1.27(0.82-1.97)$ & 0.28 \\
\hline CRT-D & $2.18(1.80-2.63)$ & $<0.001$ & $1.61(1.27-2.05)$ & $<0.001$ \\
\hline LVEF $<40 \%$ & $1.08(0.99-1.18)$ & 0.09 & & \\
\hline Hemoglobin, g/dL & $0.82(0.81-0.84)$ & $<0.001$ & $0.91(0.89-0.93)$ & $<0.001$ \\
\hline Sodium, mEq/L & $0.94(0.93-0.95)$ & $<0.001$ & $0.95(0.94-0.96)$ & $<0.001$ \\
\hline Potassium, mEq/L & $0.91(0.84-0.99)$ & 0.02 & $0.82(0.75-0.89)$ & $<0.001$ \\
\hline Diuretics use at discharge & $1.57(1.32-1.85)$ & $<0.001$ & $1.28(1.06-1.55)$ & 0.009 \\
\hline ACEI or ARB use at discharge & $0.65(0.60-0.71)$ & $<0.001$ & $0.82(0.74-0.91)$ & $<0.001$ \\
\hline$\beta$-blocker use at discharge & $0.78(0.71-0.85)$ & $<0.001$ & $0.99(0.89-1.10)$ & 0.86 \\
\hline MRA use at discharge & $1.02(0.94-1.11)$ & 0.61 & & \\
\hline Transferred to hospital or nursing facility & $1.89(1.67-2.14)$ & $<0.001$ & $1.31(1.14-1.51)$ & $<0.001$ \\
\hline
\end{tabular}

$\mathrm{HR}$, hazard ratio. Other abbreviations as in Tables 1,3.

JROADHF $(22.3 \%)$ than in JCARE-CARD (10.6\%), ATTEND (18.4\%) and WET-HF (18.6\%), except for ESC-HF-LT $(26.7 \%)$.

\section{Discussion}

This study provided important features of patients with HF patients in an era of a super-aging society in Japan. The major findings of this study were that in comparison with patients from other HF registries, our cohort was older, more likely to be female, and more likely to have HFpEF. Importantly, in-hospital and long-term outcomes were still poor in these patients.

Our previous report, JCARE-CARD, demonstrated the characteristics and outcomes of 1,677 patients with HF from 2004 to 2005, in which the patient mean age was 71 years. ${ }^{10}$ The ATTEND registry, ${ }^{11}$ which studied acute HF between 2007 and 2011, and WET-HF, ${ }^{12}$ which investigated patients with acute HF from 2011 to 2015 also revealed that patients with HF were older, 73 years and 75 years, respectively. In the JORADHF study, the mean age of patients with HF was 78 years, which was higher than that for ESC-HF-LT, ${ }^{13}$ and the highest among HF registries reported in Japan. These changes in aging in patients with $\mathrm{HF}$ reflect the advanced aging society in Japan. Life expectancy at birth increased from 79.0 years in 1990 to 83.2 years in 2015 in Japan as a result of reduced mortality and disability from most major diseases. ${ }^{14}$ Aging of patients with HF is now a critical medical and socioeconomic problem worldwide. ${ }^{2,15}$ Thus, this registry provided valuable information about patients with HF in the aging societies. The percentage of women was the highest and HFrEF was the lowest in the JROADHF compared to the other 4 registries. The ratios of all-cause death and cardiac death at 30 days, as well as all-cause death, cardiac death and hospitalization for $\mathrm{HF}$ at 1 year were the highest in JROADHF compared to other previous Japanese registries. Patients from the ESC-HF-LT were younger by 8.6 years and had more HFrEF, and cardiovascular death at 1 year was equivalent. These findings suggest that $\mathrm{HF}$ is still a frequent cause of hospitalization with high morbidity and mortality in Japan, even with the advances of pharmacological and non-pharmacological treatments.

Due to the aging of patients with HF, the prevalence of HFpEF has increased over the past decades and continues to increase. ${ }^{16-18}$ In JROADHF, mean LVEF was higher and the prevalence of HFrEF was lower than those in other registries (Table 5). Therefore, the lower prescription rate of ACEIs or ARB, and $\beta$-blockers in the JROADHF patients compared with other registries could be due to the 
lower prevalence of HFrEF (Table 5) because these drugs were recommended for HFrEF, but not for HFpEF. In contrast, the mortalities were worse in the JROADHF than in other registries (Table 5), even though the prevalence of HFpEF was higher and mortality was generally lower than for those with HFrEF, which might be due to the older age of patients (78 vs. 69-75 years). Another characteristic of JROADHF compared to other registries was the difference in etiology of HF; ischemic etiology was less, whereas arrhythmic etiology accounted for $17.3 \%$ of cases as a major cause of HF in this registry. Incidentally, atrial fibrillation $(\mathrm{AF})$ as a comorbidity was higher among other representative registries except for WET-HF, a registry undertaken in a similar era in Japan as JROADHF. Because the incidence and prevalence of AF increases with age, ${ }^{19,20}$ the alteration in the proportion of etiology will be

\begin{tabular}{|c|c|c|c|c|c|}
\hline Name & JCARE-CARD & ATTEND & WET-HF & ESC-HF-LT (AHF) & JROADHF \\
\hline Year & 2004-2005 & 2007-2011 & 2011-2015 & 2011-2015 & 2013 \\
\hline Number of patients & 1,677 & 4,842 & 2,551 & 6,629 & 13,238 \\
\hline Age & $70.7 \pm 13.5$ & $73.0 \pm 13.8$ & $75.0 \pm 13.0$ & $69.4 \pm 13.0$ & $78.0 \pm 12.5$ \\
\hline$>75$ years, $\%$ & - & - & - & 34.0 & 68.9 \\
\hline Male & 59.4 & 58.0 & 59.0 & 63.0 & 52.8 \\
\hline NYHA functional class III-IV & 88.5 & - & - & 85.2 & 85.2 \\
\hline $\mathrm{BMI}, \mathrm{kg} / \mathrm{m}^{2}$ & - & - & $23.3 \pm 4.6$ & $28.7 \pm 5.4$ & $22.9 \pm 4.6$ \\
\hline SBP, $\mathrm{mmHg}$ & $134.3 \pm 30.3$ & $145.5 \pm 36.7$ & $141.0 \pm 34.0$ & $133.5 \pm 28.2$ & $139.9 \pm 33.0$ \\
\hline Heart rate, beats/min & $87.8 \pm 24.4$ & $98.6 \pm 29.1$ & $94.0 \pm 29.0$ & $88.0[73-104]$ & $91.9 \pm 25.9$ \\
\hline LVEF, \% & $42.5 \pm 17.9$ & - & $45.0 \pm 15.0$ & $39.2 \pm 14.5$ & $46.7 \pm 17.3$ \\
\hline LVEF $<40 \%$ & - & 53.4 & 41.0 & 53.2 & 37.4 \\
\hline \multicolumn{6}{|l|}{ Etiologies, \% } \\
\hline Ischemic & 34.0 & 31.1 & 29 & 56.5 & 26.7 \\
\hline Hypertensive & 26.4 & 17.7 & - & 8.2 & 16.5 \\
\hline Valvular & 28.1 & 19.4 & - & 11.8 & 18.5 \\
\hline Cardiomyopathic & 17.0 & 12.7 & - & 13.6 & 12.4 \\
\hline Arrhythmia & - & - & - & - & 17.3 \\
\hline \multicolumn{6}{|l|}{ Comorbidities, \% } \\
\hline Hypertension & 52.1 & 69.4 & 71 & 65.6 & 71.2 \\
\hline Diabetes mellitus & 29.8 & 33.8 & 36 & 39.1 & 34.3 \\
\hline Dyslipidemia & 25.8 & 36.6 & 40 & - & 30.1 \\
\hline CKD & 11.3 & - & - & 26.3 & 38.9 \\
\hline Stroke & 16.3 & 14.0 & 14 & 12.0 & 13.7 \\
\hline Atrial fibrillation & 34.9 & 39.6 & 47 & 30.3 & 43.1 \\
\hline COPD & 5.8 & 9.5 & 6 & 19.1 & 6.4 \\
\hline \multicolumn{6}{|l|}{ Medical history, \% } \\
\hline $\mathrm{PCl}$ & 18.9 & - & & 20.2 & 17.5 \\
\hline CABG & 9.3 & - & & 9.5 & 6.8 \\
\hline Pacemaker & 1.3 & 6.6 & & 6.0 & 7.7 \\
\hline ICD & 2.2 & 3.4 & & 5.1 & 1.3 \\
\hline CRT & 1.7 & 2.3 & & 3.6 & 2.5 \\
\hline Hemodialysis & - & 1.6 & & - & 2.5 \\
\hline De novo ADHF & 50.0 & 63.8 & 70 & 69.7 & 65.3 \\
\hline \multicolumn{6}{|l|}{ Laboratory data } \\
\hline Hemoglobin, g/dL & $12.4 \pm 4.5$ & $12.0 \pm 2.6$ & $11.9 \pm 2.3$ & $12.7[11.2-14.1]$ & $11.6 \pm 2.4$ \\
\hline Serum creatinine, $\mathrm{mg} / \mathrm{dL}$ & $1.3 \pm 1.0$ & $1.4 \pm 1.6$ & $1.5 \pm 1.6$ & $1.2[0.9-1.5]$ & $1.1[0.8-1.6]$ \\
\hline $\mathrm{BUN}, \mathrm{mg} / \mathrm{dL}$ & $26.2 \pm 16.7$ & $27.8 \pm 26.0$ & $27.6 \pm 17.0$ & - & $23.9[17.4-34.7]$ \\
\hline Serum sodium, mEq/L & $139.7 \pm 4.6$ & $139.3 \pm 4.4$ & $139.2 \pm 4.3$ & $139[135-141]$ & $139.2 \pm 4.8$ \\
\hline BNP & $878 \pm 929$ & $707[362-1,284]$ & $676[351-1,221]$ & $765[355-1,398]$ & $697[376-1,309]$ \\
\hline \multicolumn{6}{|l|}{ Medication at admission, $\%$} \\
\hline Diuretics & 54.4 & 45.0 & - & - & 54.9 \\
\hline ACEls & 26.5 & 14.4 & - & - & 14.1 \\
\hline ARB & 28.9 & 34.5 & - & - & 33.5 \\
\hline ACEls or ARB & 51.2 & - & - & - & 46.2 \\
\hline$\beta$-blockers & 22.3 & 35.0 & - & - & 36.3 \\
\hline MRA & 24.1 & 20.0 & - & - & 21.6 \\
\hline
\end{tabular}

(Table 5 continued the next page.) 


\begin{tabular}{|c|c|c|c|c|c|}
\hline $\begin{array}{c}\text { Name } \\
\text { Intravenous drugs, \% }\end{array}$ & JCARE-CARD & ATTEND & WET-HF & ESC-HF-LT (AHF) & JROADHF \\
\hline Diuretics & - & 76.3 & 67.8 & 81.7 & 75.6 \\
\hline Vasodilators & 60.1 & 78.3 & 64.7 & 21.2 & 64.5 \\
\hline Inotropes & 40.8 & 18.5 & 16.8 & 12.3 & 21.2 \\
\hline \multicolumn{6}{|c|}{ Mechanical support/interventions, \% } \\
\hline Intubation & 8.9 & 7.5 & 4.9 & - & 3.0 \\
\hline NPPV & - & 24.4 & 22.2 & - & 16.5 \\
\hline Coronary angiography & - & - & - & 20.8 & 20.4 \\
\hline $\mathrm{IABP}$ & 1.0 & 2.5 & 2.3 & 1.1 & 0.8 \\
\hline PCPS & 0.2 & 0.7 & 0.5 & - & 0.1 \\
\hline $\mathrm{PCl}$ & 3.9 & 8.0 & 4.5 & - & 0.2 \\
\hline CABG & 1.0 & 1.3 & 2.0 & - & 0.2 \\
\hline $\mathrm{PCl} / \mathrm{CABG}$ & - & & - & 9.8 & 0.4 \\
\hline Pacemaker & 4.3 & 3.8 & & & 0.8 \\
\hline ICD & - & 2.6 & 1.3 & 9.6 & 0.0 \\
\hline CRT & - & 2.3 & & & 0.6 \\
\hline CRT-P & - & - & 0.5 & 1.9 & 0.2 \\
\hline CRT-D & - & - & & 6.8 & 0.4 \\
\hline \multicolumn{6}{|l|}{ Medication at discharge, $\%$} \\
\hline Diuretics & 78.8 & 82.0 & - & 83.9 & 89.9 \\
\hline ACEls & 36.9 & 30.6 & - & - & 30.1 \\
\hline ARB & 47.5 & 46.0 & - & - & 38.8 \\
\hline ACEls or ARB & 79.0 & - & - & 77.0 & 65.6 \\
\hline$\beta$-blockers & 49.8 & 67.4 & - & 72.6 & 63.0 \\
\hline MRA & 43.8 & 50.0 & - & 53.9 & 50.6 \\
\hline \multicolumn{6}{|l|}{ In-hospital outcomes } \\
\hline $\begin{array}{l}\text { Length of hospital stay, median } \\
\text { [IQR] days }\end{array}$ & 15 & 21 [14-32] & 14 [9-22] & $7[4-11]$ & $18[12-28]$ \\
\hline In-hospital all-cause death & - & 6.4 & 4.7 & 5.5 & 7.7 \\
\hline In-hospital cardiac death & - & 4.5 & 3.1 & 4.4 & 5.9 \\
\hline Follow-up period & $2.3 \pm 0.7$ & - & - & $1.0[0.8-1.1]$ & $4.3[3.8-4.7]$ \\
\hline 30-day all-cause death & 2.6 & 3.9 & 3.3 & - & 6.2 \\
\hline 30-day cardiac death & 0.2 & 3.0 & 2.2 & - & 4.8 \\
\hline 1-year all-cause death & 10.6 & 18.4 & 18.6 & 26.7 & 22.3 \\
\hline 1-year cardiac death & 6.9 & 11.5 & 10.1 & 14.3 & 14.2 \\
\hline 30-day hospitalization for HF & 4.4 & 4.6 & 5.2 & - & 3.0 \\
\hline 1-year hospitalization for HF & 26.8 & 24.4 & 27.1 & 25.9 & 29.4 \\
\hline
\end{tabular}

JROADHF data are the same as presented in Table 1. ADHF, acute decompensated heart failure; IQR, interquartile range; NPPV, non-invasive positive pressure ventilation. Other abbreviations as in Table 1.

explained, at least in part, by aging of patients with $\mathrm{HF}$ in JROADHF. Further detailed subgroup analyses, including LVEF categories and comorbidities, are necessary to provide new evidence peculiar to the elderly patients from this database.

Multivariable logistic regression analysis revealed that age, blood pressure, NYHA functional class, aortic valve stenosis, history of hypertension, chronic kidney disease, stroke, albumin level, BNP level at admission, and $\beta$-blocker use at admission were independent predictors of in-hospital death. The present study identified aortic valve stenosis as an independent predictor, which has not been reported previously as an independent predictor ${ }^{21-23}$ because most previous HF registries or trials probably excluded valvular heart disease. Primarily, the prevalence of valvular heart diseases, especially aortic valve stenosis, increased with age. Aortic valve stenosis was diagnosed when it was considered to be hemodynamically significant by the investigator at each hospital. The prevalence of aortic valve stenosis as a comorbidity among patients hospitalized due to $\mathrm{HF}$ was $8.9 \%$. Acute $\mathrm{HF}$ was known to be associated with extremely high mortality in patients with aortic valve stenosis. ${ }^{\mathbf{2 3} 24}$ Early recognition and intervention for valvular heart disease is important.

In addition, age, gender, blood pressure, NYHA functional class, prior HF hospitalization, ischemic heart disease, aortic valve stenosis, hypertension, chronic kidney disease, ventricular arrhythmia, CRT-D, hemoglobin level, sodium level, potassium level, diuretics use, and ACEI/ ARB use at discharge, transferred to hospital or nursing facilities were independently associated with cardiovascular death during the follow up after discharge, which is consistent with findings from previous reports. ${ }^{13,25}$ The use of $\beta$-blockers was associated with long-term mortality, as assessed by univariate analysis, but not by multivariate analysis. Therefore, $\beta$-blocker use at discharge might be confounding with other variables, including ACEI or ARB use, which could not be confirmed in the present analysis. 
In our multivariable logistic regression or Cox regression analysis, AF was not an independent risk factor either in in-hospital or cardiovascular death in JROADHF. The prognostic effect of atrial fibrillation has been reported to be controversial. Several studies reported that AF was associated with a worse outcome for patients with $\mathrm{HF}, \mathbf{2 6 , 2 7}$ whereas other studies found that AF was not an independent predictor of mortality. ${ }^{28,29}$ Our results were consistent with previous analysis using JCARE-CARD, a registry for hospitalized patients with HF in Japan, in which there was no difference in the long-term outcome of HF patients with and without AF. ${ }^{30}$

'Transfer to hospital or nursing facility' was positively associated with long-term cardiovascular death, suggesting that functional activity of daily living is an important prognostic factor in patients with $\mathrm{HF}$, as previously reported..$^{31,32}$

We utilized JROAD-DPC for randomization and all the information associated with medical procedures, and clinical information including prognosis were collected from both DPC-based claim data and clinical records, which enabled us to create a large-scale, nationwide, high-accuracy, comprehensive cross-sectional and longitudinal HF database. Therefore, the present study could confirm the previous findings by using the JROADHF database, which represents the real-world clinical practice of HF in Japan.

\section{Study Limitations}

Several limitations must be acknowledged in the present study. First, since JROADHF is a retrospective study, the variables are limited to existing medical information and procedures. Meanwhile, the present study collected a wide variety of data-specific and supplementary information from medical charts and a diagnosis procedure combination system, including long-term outcomes of most patients after discharge. As a result, this registry reflects the realworld evidence of clinical practice of HF in Japan. Second, we could not enroll all the patients screened by cluster random sampling because 65 facilities did not participate. We obtained the clinical and DPC data only from the hospitals that had agreed to be included in the registry, we thus could not analyze the characteristics of excluded patients. However, the nationwide distribution of institutions and patients by regions at final enrollment did not differ from that at sampling (Figure 2). Third, we included only JCS-certified hospitals in JROADHF and also excluded hospitals with fewer than $11 \mathrm{HF}$ admissions. Therefore, the generalization of our results is limited and may not be applicable to small or non-specialized hospitals. Fourth, the data of JROADHF were collected from the records of 2013, which is not very recent, and patients with HF are older now; therefore, we needed to set a sufficient follow-up period for analysis. Fifth, subgroup analysis data according to LVEF categories were not available in the present study. These data were essential to interpret the differences in the treatments and outcomes between JROADHF and the previous registries (Table 5) because the recommended medications and estimated outcomes might differ by LVEF categories. Further analysis examining the influence of differences in LVEF categories is definitely needed. Finally, hospital factors including case volume, ${ }^{33}$ access to medical care ${ }^{34}$ or combination of scale and variations of clinical procedures ${ }^{35}$ may influence the prognosis of HF; however, we did not include these factors in this analysis, except for patients clinical factors. Further analysis is needed to determine whether these factors are the predictors of HF prognosis.

\section{Conclusions}

Based on a nationwide HF database including more than 13,000 Japanese patients, the present study clarified the characteristics and in-hospital and long-term outcomes of hospitalized patients with HF in Japan. In comparison with patients of other HF registries, our registry was older, included more females, and increased HFpEF. The incidence of HF caused by arrhythmia or valvular disease may be increased due to the aging of patients with HF. These findings will contribute to the development of effective and efficient management of $\mathrm{HF}$ in the future.

\section{One sentence summary}

A Nationwide large-scale registry showed the contemporary realworld characteristics and outcomes of hospitalized patients with heart failure in Japan.

\section{Acknowledgments}

This study was conducted on behalf of all the members and institutions of the Japanese Registry Of Acute Decompensated Heart Failure (JROADHF) registry. The participating institutions and investigators of JROADHF are listed in the Supplementary File.

The authors thank Fumika Yamashita (Kyushu University Hospital), Makoto Yokobori (Suxac Inc., Japan), Tomoe Mashiko (Suxac Inc., Japan), Go Ichien (HuBit Genomix Inc., Japan), Masako Terauchi (HuBit Genomix Inc., Japan), Mao Kakinoki (HuBit Genomix Inc., Japan), Sachiko Matsuoka (HuBit Genomix Inc., Japan), Satoko Ishihara (G-ONE Inc. Japan) and Keigo Mori (G-ONE Inc., Japan) for their valuable support of the JROADHF registry.

This work was supported by the Japan Agency for Medical Research and Development grant (19ek0210097h0003 (I.K.), 19ek0109339h0002 (H.T.), 19ek0210080h0003 (H.T.)), Grants-in-Aid for Scientific Research from the Ministry of Education, Culture, Sports, Science, and Technology of Japan (17K09582 (T.I.), 15 H04815 (H.T.)), and Health Labour Sciences Research Grant (20FC1051). The funders had no role in the design and conduct of the study or in collection, management, analysis and interpretation of the data, the preparation, review, and submission of the manuscript for publication.

\section{Disclosures}

All authors have completed and submitted the ICMJE Form for Disclosure of Potential Conflicts of Interest.

M.H. reports receipt of donations/scholarship funds from ONO PHARMACEUTICAL CO., LTD, and Daiichi-Sankyo, and a scholarship (educational) grant from Otsuka Pharmaceutical Co., Ltd., Nipro Corporation, and TERUMO CORPORATION

I.K. reports receipt of donations/scholarship funds from AstraZeneca K.K, Actelion Pharmaceuticals Japan Ltd., Astellas Pharma Inc., Amgen Astellas BioPharma K.K, ONO PHARMACEUTICAL CO., LTD., MSD K.K., Daiichi-Sankyo, Takeda Pharmaceutical Company Limited, Nippon Boehringer Ingelheim Co., Ltd, BAYER YAKUHIN, LTD, Pfizer Japan Inc., and a research grant from ONO PHARMACEUTICAL CO., LTD., Meiji Co., Ltd., and a scholarship donation from KOWA Company, Ltd, Daiichi-Sankyo, Takeda Pharmaceutical Company Limited, Mitsubishi Tanabe Pharma Corporation, TEIJIN PHARMA LIMITED, Nipro Corporation, and TERUMO CORPORATION.

H.T. reports receipt of scholarship funds from MSD, Astellas, Pfizer, Bristol-Myers Squibb, Otsuka Pharmaceutical, Daiichi-Sankyo, Mitsubishi Tanabe Pharma, Nippon Boehringer Ingelheim, Takeda Pharmaceutical, Bayer Yakuhin, Novartis Pharma, Kowa Pharmaceutical, Teijin Pharma, Medical Review Co., and the Japanese Journal of Clinical Medicine; scholarship funds from Astellas, Novartis Pharma, Daiichi-Sankyo, Takeda Pharmaceutical, Mitsubishi Tanabe Pharma, Teijin Pharma and MSD, outside of the submitted work.

T.I. endowed chair funded by Actelion Pharmaceuticals. M.H. endowed chair from Otsuka Pharmaceutical Co., Ltd., Nipro Corporation, and TERUMO CORPORATION.

H.T. received non-financial support from Actelion Pharmaceuticals, Mitsubishi Tanabe Pharma, Nippon Boehringer Ingelheim, Daiichi- 
Sankyo, IQVIA Services Japan, and Omron Healthcare Co.

T.I., I.K., H.T. are the members of Circulation Journal's Editorial Team.

\section{IRB Information}

The original study protocol was approved by the institutional review boards of Kyushu University (2019-569), the Japanese Circulation Society (2017. No3) and all of the 128 participating hospitals.

\section{Data Availability}

(1) Whether individual deidentified participant data (including data dictionaries) will be shared.

-Yes.

(2) What data in particular will be shared?

- Individual participant data of each hospital that underlie the results reported in this article, after deidentification.

(3) Whether additional, related documents will be available. - No.

(4) When the data will become available and for how long. - The data of each participating hospital will become available for use after this article is published in the journal online and for 1 year.

(5) By what access criteria will the data be shared (including with whom)?

- The data will be shared on a request basis by the investigators who participated in the JROADHF.

(6) For what types of analyses, and by what mechanism will the data be available?

- The data can be applicable to any type of analysis for academic use. Permission from the publication committee of JROADHF is necessary before publication. The data will be shared as Excel or CSV files via email.

\section{References}

1. Ambrosy AP, Fonarow GC, Butler J, Chioncel O, Greene SJ, Vaduganathan M, et al. The global health and economic burden of hospitalizations for heart failure: Lessons learned from hospitalized heart failure registries. J Am Coll Cardiol 2014; 63: $1123-1133$

2. Conrad N, Judge A, Tran J, Mohseni H, Hedgecott D, Crespillo $\mathrm{AP}$, et al. Temporal trends and patterns in heart failure incidence: A population-based study of 4 million individuals. Lancet 2018; 391: 572-580.

3. Chioncel O, Mebazaa A, Harjola VP, Coats AJ, Piepoli MF, Crespo-Leiro MG, et al; Investigators ESCHFL-TR. Clinical phenotypes and outcome of patients hospitalized for acute heart failure: The ESC Heart Failure Long-Term Registry. Eur J Heart Fail 2017; 19: $1242-1254$.

4. Tsutsui H, Tsuchihashi-Makaya M, Kinugawa S, Goto D, Takeshita A, Investigators J-C. Clinical characteristics and outcome of hospitalized patients with heart failure in Japan. Circ J 2006; 70: 1617-1623.

5. Yasuda S, Miyamoto Y, Ogawa H. Current status of cardiovascular medicine in the aging society of Japan. Circulation 2018; 138: $965-967$.

6. Shimokawa H, Miura M, Nochioka K, Sakata Y. Heart failure as a general pandemic in Asia. Eur J Heart Fail 2015; 17: 884-892.

7. Ho KK, Pinsky JL, Kannel WB, Levy D. The epidemiology of heart failure: The Framingham Study. J Am Coll Cardiol 1993; 22(4 Suppl A): $6 \mathrm{~A}-13 \mathrm{~A}$.

8. Yasunaga H, Ide H, Imamura T, Ohe K. Impact of the Japanese Diagnosis Procedure Combination-based Payment System on cardiovascular medicine-related costs. Int Heart J 2005; 46: 855-866.

9. Rothman, JK, Greenland S, Lash LT. Modern Epidemiology, $3^{\text {rd }}$ edition, Chapter 6. Philadelphia: Lippincott Williams \& Wilkins, 2012; 92-93.

10. Hamaguchi S, Kinugawa S, Tsuchihashi-Makaya M, Goto D, Yamada S, Yokoshiki H, et al. Characteristics, management, and outcomes for patients during hospitalization due to worsening heart failure-A report from the Japanese Cardiac Registry of Heart Failure in Cardiology (JCARE-CARD). J Cardiol 2013; 62: $95-101$.

11. Sato N, Kajimoto K, Keida T, Mizuno M, Minami Y, Yumino $\mathrm{D}$, et al. Clinical features and outcome in hospitalized heart failure in Japan (from the ATTEND Registry). Circ J 2013; 77: 944-951.

12. Shiraishi Y, Kohsaka S, Sato N, Takano T, Kitai T, Yoshikawa
T, et al. 9-year trend in the management of acute heart failure in Japan: A report from the National Consortium of Acute Heart Failure Registries. J Am Heart Assoc 2018; 7: e008687.

13. Crespo-Leiro MG, Anker SD, Maggioni AP, Coats AJ, Filippatos G, Ruschitzka F, et al. European Society of Cardiology Heart Failure Long-Term Registry (ESC-HF-LT): 1-year follow-up outcomes and differences across regions. Eur J Heart Fail 2016; 18: $613-625$.

14. Nomura S, Sakamoto H, Glenn S, Tsugawa Y, Abe SK, Rahman $\mathrm{MM}$, et al. Population health and regional variations of disease burden in Japan, 1990-2015: A systematic subnational analysis for the Global Burden of Disease Study 2015. Lancet 2017; 390: $1521-1538$

15. Lawson CA, Zaccardi F, Squire I, Okhai H, Davies M, Huang W, et al. Risk factors for heart failure: 20 -year population-based trends by sex, socioeconomic status, and ethnicity. Circ Heart Fail 2020; 13: e006472.

16. Owan TE, Hodge DO, Herges RM, Jacobsen SJ, Roger VL, Redfield MM. Trends in prevalence and outcome of heart failure with preserved ejection fraction. $N$ Engl J Med 2006; 355: 251 - 259.

17. Mozaffarian D, Benjamin EJ, Go AS, Arnett DK, Blaha MJ, Cushman M, et al. Heart disease and stroke statistics--2015 update: A report from the American Heart Association. Circulation 2015; 131: e29-e322.

18. Aljaroudi W, Alraies MC, Halley C, Rodriguez L, Grimm RA, Thomas JD, et al. Impact of progression of diastolic dysfunction on mortality in patients with normal ejection fraction. Circulation 2012; 125: 782-788.

19. Miyasaka Y, Barnes ME, Gersh BJ, Cha SS, Bailey KR, Abhayaratna WP, et al. Secular trends in incidence of atrial fibrillation in Olmsted County, Minnesota, 1980 to 2000, and implications on the projections for future prevalence. Circulation 2006; 114: $119-125$.

20. Lakshminarayan K, Solid CA, Collins AJ, Anderson DC, Herzog CA. Atrial fibrillation and stroke in the general medicare population: A 10-year perspective (1992 to 2002). Stroke 2006; 37: $1969-1974$.

21. Ancion A, Allepaerts S, Oury C, Gori AS, Pierard LA, Lancellotti P. Serum albumin level and hospital mortality in acute nonischemic heart failure. ESC Heart Fail 2017; 4: 138-145.

22. Abraham WT, Fonarow GC, Albert NM, Stough WG, Gheorghiade M, Greenberg BH, et al. Predictors of in-hospital mortality in patients hospitalized for heart failure: Insights from the Organized Program to Initiate Lifesaving Treatment in Hospitalized Patients with Heart Failure (OPTIMIZE-HF). J Am Coll Cardiol 2008; 52: 347-356.

23. Fonarow GC, Peacock WF, Phillips CO, Givertz MM, Lopatin M, ADHERE Scientific Advisory Committee and Investigators. Admission B-type natriuretic peptide levels and in-hospital mortality in acute decompensated heart failure. $\mathrm{J} \mathrm{Am} \mathrm{Coll} \mathrm{Cardiol}$ 2007; 49: 1943-1950.

24. Nagao K, Taniguchi T, Morimoto T, Shiomi H, Ando K, Kanamori N, et al. Acute heart failure in patients with severe aortic stenosis: Insights from the CURRENT AS Registry. Circ J 2018; 82: 874-885.

25. Motiejunaite J, Akiyama E, Cohen-Solal A, Maggioni AP, Mueller C, Choi DJ, et al. The association of long-term outcome and biological sex in patients with acute heart failure from different geographic regions. Eur Heart J 2020; 41: 1357-1364.

26. Mountantonakis SE, Grau-Sepulveda MV, Bhatt DL, Hernandes AF, Peterson ED, Fonarow GC. Presence of atrial fibrillation is independently associated with adverse outcomes in patients hospitalized with heart failure: An analysis of get with the guidelines-Heart Failure. Circ Heart Fail 2012; 5: 191 - 201.

27. Dries DL, Exner DV, Gersh BJ, Domanski MJ, Waclawiw MA, Stevenson LW. Atrial fibrillation is associated with an increased risk for mortality and heart failure progression in patients with asymptomatic and symptomatic left ventricular systolic dysfunction: A retrospective analysis of the SOLVD trials. J Am Coll Cardiol 1998; 32: 695-703.

28. Mahoney P, Kimmel S, DeNofrio D, Wahl P, Loh E. Prognostic significance of atrial fibrillation in patients at a tertiary medical center referred for heart transplantation because of severe heart failure. Am J Cardiol 1999; 83: 1544-1547.

29. Crijns HJ, Tjeerdsma G, de Kam PJ, Boomsma F, van Gelder IC, van den Berg MP, et al. Prognostic value of the presence and development of atrial fibrillation in patients with advanced chronic heart failure. Eur Heart J 2000; 21: 1238-1245.

30. Hamaguchi S, Yokoshiki H, Kinugawa S, Tsuchihashi-Makaya M, Yokota T, Takeshita A, et al. Effects of atrial fibrillation on 
long-term outcomes in patients hospitalized for heart failure in Japan: A report from the Japanese Cardiac Registry of Heart Failure in Cardiology (JCARE-CARD). Circ J 2009; 73: 2084-2090.

31. Dunlay SM, Manemann SM, Chamberlain AM, Cheville AL, Jiang R, Weston SA, et al. Activities of daily living and outcomes in heart failure. Circ Heart Fail 2015; 8: 261-267.

32. Uemura Y, Shibata R, Takemoto K, Koyasu M, Ishikawa S, Murohara T, et al. Prognostic impact of the preservation of activities of daily living on post-discharge outcomes in patients with acute heart failure. Circ J 2018; 82: 2793-2799.

33. Yasuda S, Nakao K, Nishimura K, Miyamoto Y, Sumita Y, Shishido T, et al. The current status of cardiovascular medicine in Japan: Analysis of a large number of health records from a Nationwide Claim-Based Database, JROAD-DPC. Circ J 2016; 80: $2327-2335$.
34. Gamble JM, Eurich DT, Ezekowitz JA, Kaul P, Quan H, McAlister FA. Patterns of care and outcomes differ for urban versus rural patients with newly diagnosed heart failure, even in a universal healthcare system. Circ Heart Fail 2011; 4: 317-323.

35. Kaku H, Funakoshi K, Ide T, Fujino T, Matsushima S, Ohtani $\mathrm{K}$, et al. Impact of hospital practice factors on mortality in patients hospitalized for heart failure in Japan: An Analysis of a large number of health records from a nationwide claims-based database, the JROAD-DPC. Circ J 2020; 84: 742-753.

\section{Supplementary Files}

Please find supplementary file(s); http://dx.doi.org/10.1253/circj.CJ-20-0947 